\title{
Quality Gaps and Comparative Effectiveness in Lung Cancer Staging
}

\section{The Impact of Test Sequencing on Outcomes}

\author{
Francisco A. Almeida, MD, FCCP; Roberto F. Casal, MD; Carlos A. Jimenez, MD, FCCP; \\ George A. Eapen, MD; Mateen Uzbeck, MBBS; Mona Sarkiss, MD; David Rice, MD; \\ Rodolfo C. Morice, MD, FCCP; and David E. Ost, MD, MPH, FCCP
}

\begin{abstract}
Background: Evidence-based guidelines recommend mediastinal sampling as the first invasive test in patients with suspected lung cancer and mediastinal adenopathy. The goal of this study was to assess practice patterns and outcomes of diagnostic strategies in this patient population.

Methods: We conducted a retrospective analysis of all patients in 2009 who had mediastinal adenopathy without distant metastatic disease to determine whether guideline-consistent care was delivered. Guideline-consistent care was defined as mediastinal lymph node sampling being performed as part of the first invasive procedure.

Results: One hundred thirty-seven patients were included. Guideline-consistent care was provided in 30 cases $(22 \%)$. Patients receiving guideline-consistent care had fewer invasive tests than patients with guideline-inconsistent care $(1.3 \pm 0.5$ tests/patient vs $2.3 \pm 0.5$ tests/patient, respectively; $P<.0001)$ and fewer complications $(0$ of $30,0 \%$ vs 18 of $108,17 \% ; P=.01)$. Most of the complications (16 of 18) were related to CT image-guided needle biopsy. Endobronchial ultrasound-guided transbronchial needle aspiration (EBUS-TBNA) was sufficient to guide treatment decisions without any other invasive tests in 88 patients $(64 \%)$. Although not all the complications and costs due to $\mathrm{CT}$ image-guided biopsies could have been avoided, roughly two-thirds could have been eliminated by just changing the testing sequence.

Conclusion: Quality gaps in lung cancer staging in patients with mediastinal adenopathy are common and lead to unnecessary testing and increased complications. In patients with suspected lung cancer without distant metastatic disease with mediastinal adenopathy, EBUS-TBNA should be the first test.

CHEST 2013; 144(6):1776-1782
\end{abstract}

Abbreviations: EBUS = endobronchial ultrasound; EBUS-TBNA = endobronchial ultrasound-guided transbronchial needle aspiration; EGFR = epidermal growth factor receptor; TBNA = transbronchial needle aspiration

A ccurate staging is critical to the effective treatment of lung cancer. For patients without evidence of distant metastatic disease, assessment of the mediastinal lymph nodes is important, since the status of these nodes will determine treatment. ${ }^{1,2}$ Clinical assessment of the mediastinal lymph nodes is guided by noninvasive imaging modalities, such as CT scan and PET scan. The median sensitivity and specificity of CT scanning for identifying malignant involvement of mediastinal nodes is $61 \%$ and $79 \%$, respectively. ${ }^{3}$ The sensitivity of PET scanning is conditional upon whether the nodes are enlarged on CT scan. If the lymph nodes are enlarged on CT scan, the median sensitivity and specificity of PET scan is $100 \%$ and $78 \%$, respectively. If the lymph nodes are not enlarged on CT scan, the median sensitivity and specificity of PET scan is $82 \%$ and $93 \%$, respectively. ${ }^{3}$ Because of the limited predictive value of both CT scanning and PET scanning, current guidelines recommend that patients with mediastinal adenopathy by CT scan or PET scan undergo lymph node sampling to ensure accurate staging., ${ }^{1,2}$

For editorial comment see page 1747

In patients with lung masses and evidence of mediastinal adenopathy without evidence of distant metastatic disease, a common clinical question is whether to perform biopsy of the peripheral lung lesions or the lymph nodes first. When there are discrete enlarged lymph nodes on CT scan or PET scan without direct 
infiltration of the mediastinum, the 2007 American College of Chest Physicians evidence-based guidelines recommend mediastinal lymph node sampling as the first procedure, since this can both diagnose and stage disease with one procedure..$^{2,-6}$ This is consistent with other guidelines on lung cancer that recommend thinking carefully before performing a test that gives only a diagnosis when information on staging is also needed. . $^{-9}$

However, there may be large gaps between what is recommended in guidelines and what is actually done in practice. Indeed, previous studies have identified practice gaps in lung cancer staging. ${ }^{10-15}$ Studies of mediastinoscopy and conventional transbronchial needle aspiration (TBNA) found that these techniques have been underused to stage the mediastinum. ${ }^{10-15}$ Endobronchial ultrasound (EBUS)-guided TBNA (EBUSTBNA) has been introduced and has been shown to be superior to conventional TBNA and at least equivalent to mediastinoscopy. ${ }^{16-19}$ However, EBUS-TBNA is not always available.

But aside from whether these techniques are used, there has been little study of how these tests are used. More specifically, there have been no studies evaluating when in the testing sequence mediastinal sampling techniques are used. According to evidence-based guidelines, mediastinal sampling should be the first invasive test for both diagnosis and staging in patients with suspected lung cancer and mediastinal adenopathy. However, in our experience many physicians choose to perform biopsy of peripheral lesions first and only later sample the mediastinum.

Therefore, the goal of this study was to assess practice patterns and outcomes of diagnostic strategies in patients with suspected lung cancer with evidence of possible intrathoracic nodal disease by CT scan or PET scan. We hypothesized that practice gaps in lung

Manuscript received December 20, 2012; revision accepted April 15, 2013.

Affiliations: From the Department of Pulmonary, Allergy, and Critical Care Medicine (Dr Almeida), Cleveland Clinic, Cleveland, $\mathrm{OH}$; the Department of Pulmonary and Critical Care Medicine (Dr Casal), Michael E. DeBakey Veterans Affairs Medical Center, Baylor College of Medicine, Houston, TX; the Department of Pulmonary Medicine (Drs Jimenez, Eapen, Morice, and Ost), the Department of Anesthesia (Dr Sarkiss), and the Department of Thoracic Surgery (Dr Rice), The University of Texas MD Anderson Cancer Center, Houston, TX; and The Department of Pulmonary Medicine (Dr Uzbeck), Our Lady of Lourdes and Beaumont Hospitals, Dublin, Ireland.

Funding/Support: The authors have reported to CHEST that no funding was received for this study.

Correspondence to: David E. Ost, MD, MPH, FCCP, The University of Texas MD Anderson Cancer Center, Department of Pulmonary Medicine Unit 1462, 1515 Holcombe Blvd, Houston, TX 77030; e-mail: dost@mdanderson.org

(C) 2013 American College of Chest Physicians. Reproduction of this article is prohibited without written permission from the American College of Chest Physicians. See online for more details. DOI: 10.1378/chest.12-3046 cancer staging persist, particularly when it comes to the proper sequencing of tests, and that improper test sequencing would lead to unnecessary tests and complications.

\section{Materials AND Methods}

We conducted a retrospective analysis of consecutive patients with suspected or confirmed lung cancer, stage T1-3N1-3M0 by CT scan and PET scan, referred to thoracic surgery or pulmonary medicine for evaluation of mediastinal adenopathy between January and December 2009. This included patients in whom the purpose of the evaluation was to establish a diagnosis, stage the mediastinum, or both. This study was approved by institutional review board 4, DR09-0101, and a waiver of informed consent was obtained. Patients undergoing EBUS-TBNA for restaging, those with a contralateral lesion suspicious for a second primary lung cancer, those with a history of another thoracic tumor within the prior 5 years, or those with mesothelioma were excluded.

CT scanning and PET scanning were used to arrive at a presumptive clinical-radiographic TNM stage. Lymph nodes were considered positive if they were $>1 \mathrm{~cm}$ in short axis on CT scan or had a standard uptake value $>2.5$ on PET scan. If there was discordance between CT scans and PET scans as to the lymph node stage, the higher stage was always chosen..$^{20}$ Invasive procedure results and complications were recorded. Invasive procedures were defined as CT image-guided needle biopsy, mediastinoscopy or Chamberlain procedures, bronchoscopy, endoscopic ultrasonography, thoracotomy, or video-assisted thoracic surgery.

Data on bronchoscopy were obtained from a prospectively collected bronchoscopy registry. ${ }^{21-23}$ EBUS-TBNA (XBF-UC 160F, Olympus Corporation) was performed under general anesthesia beginning on the side contralateral to the mass. All N3 nodes underwent imaging and sampling, followed by $\mathrm{N} 2$ and $\mathrm{N} 1$ nodes. All lymph nodes measuring $\geq 0.50 \mathrm{~cm}$ by EBUS were sampled, even if they were normal on CT scans and PET scans. Rapid on-site evaluation was used.

The primary outcome was whether guideline-consistent care was delivered. Guideline-consistent care was defined as mediastinal lymph node sampling being performed as part of the first invasive procedure. Procedures defined as adequate for lymph node sampling included conventional bronchoscopic TBNA, EBUSTBNA, endoscopic ultrasound with lymph node sampling, or mediastinoscopy. If bronchoscopy without TBNA or a CT image-guided biopsy of peripheral lesions was performed as the first invasive test, it was classified as guideline inconsistent.

Secondary outcomes included the total number of invasive procedures, complications, and whether EBUS-TBNA was sufficient to provide all the necessary information to guide treatment. Complications included occurrence of any pneumothorax, pneumothorax requiring chest tube placement, and hypoxemia. Hypoxemia was defined as an oxygen saturation $<90 \%$ despite supplemental oxygen lasting $\geq 60 \mathrm{~s}$. EBUS-TBNA was considered as sufficient when a specific tissue diagnosis was obtained from lymph nodes and no additional invasive tests were required. If EBUS-TBNA demonstrated adequate lymph node tissue but no evidence of cancer involvement, and bronchoscopy provided a specific tissue diagnosis through other means (ie, transbronchial biopsy, brush, or lavage), and the patient went on to curative surgery or definitive treatment (ie, radiation) without any other invasive tests, then this was considered as sufficient. If EBUS-TBNA provided a specific diagnosis other than cancer (ie, sarcoidosis) such that no further invasive testing was required and the patient did not have cancer during a 2-year follow-up, this was also considered sufficient. If EBUS-TBNA and bronchoscopy failed to provide a specific 
diagnosis, or if additional invasive tests had to be performed for any reason, it was classified as insufficient.

\section{Statistical Analysis}

Characteristics of patients and outcomes were compared using $\chi^{2}$ test for categorical variables and $t$ tests for continuous normally distributed variables. We used two-sided tests and a $P$ value $<.05$ to define statistical significance. No adjustments were made for multiple comparisons. All data were analyzed with STATA/IC 12.1 (StataCorp LP).

\section{RESULTS}

A total of 137 patients with suspected or proven lung cancer with T1-3N1-3M0 disease treated at MD Anderson Cancer Center were included (Table 1). Sampling of the mediastinum was the first invasive diagnostic test, as recommended by guidelines, in only 30 cases (22\%). In 63 cases (46\%), CT image-guided needle biopsy was the first test, whereas in 44 cases (32\%), bronchoscopy without TBNA was the first test.

Patients who had guideline-consistent care had fewer invasive tests than patients with guideline-inconsistent care ( $1.3 \pm 0.5$ tests/patients vs $2.3 \pm 0.5$ tests/patient, respectively; $P<.0001$ ). This was because patients in the guideline-consistent group had fewer CT imageguided needle biopsies $(P<.001)$ and bronchoscopies without TBNA $(P<.001)$ during their diagnostic evaluation (Table 2 ). As a result, patients with guidelineconsistent care had fewer complications than patients with guideline-inconsistent care ( 0 of $30,0 \%$ vs 18 of $108,17 \% ; P=.01$ ) (Table 3). Most of these complications (16 of 18) were related to CT image-guided needle biopsy.

EBUS-TBNA was sufficient to guide treatment decisions without any other invasive tests in 88 of 137 patients (64\%). EBUS-TBNA established a specific diagnosis of lymph node involvement in 67 patients (49\%). In another 21 patients (15\%), EBUS-TBNA demonstrated adequate lymph node sampling but did not establish evidence of lymph node involvement, but other bronchoscopic methods were able to establish a specific tissue diagnosis of the peripheral lesion, and no other invasive tests were required (Table 4).

To assess the relationship between physician specialty and practice patterns, we divided patients into those who had their entire workup done at our institution $(\mathrm{n}=41)$ and those who had some of it done prior to referral $(n=96)$. This was because referral patterns at our institution are different than most hospitals, with patients with untreated lung cancer seeing a specialist as their initial point of contact. Among the 41 patients with their entire workup done at our institution, $29(71 \%)$ had EBUS-TBNA performed as their first test, in accordance with guidelines. EBUS-TBNA provided a specific diagnosis such that no other tests were required in 22 of these 29 patients (76\%). Among
Table 1-Patient Characteristics

\begin{tabular}{|c|c|}
\hline Characteristics & Patients \\
\hline Age, mean $\pm \mathrm{SD}, \mathrm{y}$ & $65 \pm 11$ \\
\hline \multicolumn{2}{|l|}{ Sex } \\
\hline Male & 71 \\
\hline Female & 66 \\
\hline \multicolumn{2}{|l|}{ ECOG performance status } \\
\hline 0 & 27 \\
\hline 1 & 101 \\
\hline$\geq 2$ & 9 \\
\hline \multicolumn{2}{|l|}{ T stage by CT scan and PET scan } \\
\hline $1 \mathrm{~A}$ & $15(11)$ \\
\hline $1 \mathrm{~B}$ & $16(12)$ \\
\hline $2 \mathrm{~A}$ & $41(30)$ \\
\hline $2 \mathrm{~B}$ & $19(14)$ \\
\hline 3 & $46(34)$ \\
\hline \multicolumn{2}{|l|}{$\mathrm{N}$ stage by CT-PET scan } \\
\hline 1 & $25(18)$ \\
\hline 2 & $71(52)$ \\
\hline 3 & $41(30)$ \\
\hline \multicolumn{2}{|l|}{ Final diagnosis } \\
\hline \multicolumn{2}{|l|}{ Lung cancer } \\
\hline Adenocarcinoma & $41(30)$ \\
\hline Squamous & $38(28)$ \\
\hline NSCLC & $35(26)$ \\
\hline Small cell & $6(4)$ \\
\hline Large cell neuroendocrine & $3(2)$ \\
\hline Carcinoid & $1(1)$ \\
\hline \multicolumn{2}{|l|}{ Solid tumor metastases ${ }^{a}$} \\
\hline Metastatic cervical cancer & $1(1)$ \\
\hline Metastatic colon cancer & $1(1)$ \\
\hline Metastatic esophageal cancer & $1(1)$ \\
\hline Metastatic hepatocellular carcinoma & $1(1)$ \\
\hline Metastatic squamous cell of lip & $1(1)$ \\
\hline \multicolumn{2}{|l|}{ Lymphomas } \\
\hline Hodgkin’s lymphoma & $1(1)$ \\
\hline Non-Hodgkin’s lymphoma & $1(1)$ \\
\hline Mycobacterium lentiflavum & $1(1)$ \\
\hline Pneumoniab & $2(1)$ \\
\hline Sarcoidosis & $3(2)$ \\
\hline
\end{tabular}

Data presented as No. or No. (\%) unless otherwise noted. ECOG $=$ Eastern Cooperative Oncology Group; NSCLC = non-small cell lung cancer.

aPatients with prior solid tumors without evidence of disease recurrence within the past $5 \mathrm{y}$.

bOne case was histoplasmosis, the other was a granulomatous inflammatory pattern presumed to have been fungal, treated with itraconazole. Radiograph cleared, but no definitive culture results; followed for $2 \mathrm{y}$.

the 96 patients who had their first invasive tests performed prior to arrival at our institution, EBUS-TBNA provided a tissue diagnosis and staging information such that any previously performed biopsies of the peripheral lesion were unnecessary in 61 cases (64\%). In guideline-inconsistent cases from outside of our institution, the most common specialties involved in ordering CT image-guided biopsies were pulmonary, internal medicine, and thoracic surgery; in guidelineinconsistent cases within our institution, the most common specialties were oncology and thoracic surgery $(P<.001)$ (Table 5). 
Table 2-Other Invasive Diagnostic Tests Performed

\begin{tabular}{|c|c|c|c|}
\hline Procedure & $\begin{array}{l}\text { Guideline-Consistent Care Mediastinal } \\
\text { Sampling First, No. }(\%)(\mathrm{n}=30)\end{array}$ & $\begin{array}{l}\text { Guideline-Inconsistent Care Mediastinal } \\
\text { Sampling 2nd+, No. (\%) }(\mathrm{n}=107)\end{array}$ & $P$ Value \\
\hline CT image-guided needle biopsy & $5(17)$ & $75(70)$ & $<.001$ \\
\hline Bronchoscopy without TBNA ${ }^{a}$ & $1(3)$ & $47(44)$ & $<.001$ \\
\hline Mediastinoscopy & $2(7)^{\mathrm{b}}$ & $11(10)$ & .73 \\
\hline
\end{tabular}

Patients could have more than one invasive diagnostic test. EBUS-TBNA = endobronchial ultrasound-guided transbronchial needle aspiration; TBNA $=$ transbronchial needle aspiration.

aThree patients had two flexible bronchoscopies prior to EBUS-TBNA, so a total of 50 bronchoscopies without TBNA were performed in 48 patients.

bOne patient had mediastinoscopy on the same day as EBUS-TBNA as part of a study protocol. Both EBUS-TBNA and mediastinoscopy were negative and patient went for curative surgery subsequently. One patient had a Chamberlain procedure first followed by EBUS-TBNA later.

Epidermal growth factor receptor (EGFR) mutation status was ordered using EBUS samples in 18 patients. Mutation status was positive in two, negative in 13, and the quantity was insufficient in three (17\%). EGFR mutation status was ordered on 23 CT image-guided biopsy samples. Mutation status was positive in five, negative in 12 , and the quantity was insufficient in six (26\%).

\section{Discussion}

Quality gaps are defined as the difference between the health-care processes or outcomes observed in practice and those potentially obtainable on the basis of current knowledge. ${ }^{24,25}$ There are substantial data that there are wide gaps between evidence-based guidelines and what is done in clinical practice..$^{24,25}$ Our findings indicate that there is a significant quality gap in the diagnostic evaluation of patients with lung cancer. Specifically, we found that in patients with T1-3N1$3 \mathrm{M} 0$ disease, mediastinal lymph node sampling was performed first as per guidelines in only $22 \%$ of patients. In $78 \%$ of patients, a biopsy of the peripheral mass was performed first. The consequence of these peripheral mass biopsies was that patients underwent more tests $(1.3$ tests/patient vs 2.3 tests/patient, $P<.0001)$ and had more complications ( $0 \%$ vs $17 \%, P=.01)$.
Most of these additional complications were due to CT image-guided biopsies. We found that EBUS-TBNA was sufficient to provide both staging and diagnostic information in $64 \%$ of patients. So although not all of the complications and costs due to CT image-guided biopsies could have been avoided, roughly two-thirds could have been eliminated by just changing the testing sequence.

At first glance, this may seem paradoxical, since the sensitivity of CT image-guided biopsy is $90 \%$, whereas the sensitivity of bronchoscopy for peripheral lesions is $34 \%$ to $63 \%$, depending on the size of the lesion. ${ }^{6}$ However, although it is true that CT image-guided biopsy provides a diagnosis more frequently, treatment decisions require both staging information and a tissue diagnosis. CT image-guided biopsy can only provide a tissue diagnosis, whereas EBUS-TBNA provides both staging and diagnostic information. Physicians often approach this problem by thinking in seriesdiagnose first, and then stage. This linear thinking has logical appeal and has been ingrained in training programs. It is even reflected and reinforced by the language we use, since the problem is often referred to as "diagnosis and staging," implying somewhat that diagnosis comes first. But in this case it is more effective to conceptualize the problem as a unified whole

Table 3-Complications Due to Various Procedures

\begin{tabular}{|c|c|c|}
\hline Procedure & $\begin{array}{l}\text { Guideline-Consistent Care Mediastinal Sampling } \\
\text { First }(\mathrm{n}=30)\end{array}$ & $\begin{array}{l}\text { Guideline-Inconsistent Care Mediastinal } \\
\text { Sampling } 2 \mathrm{nd}+(\mathrm{n}=107)\end{array}$ \\
\hline \multicolumn{3}{|l|}{ CT image-guided needle biopsy outcomes } \\
\hline No pneumothorax & 5 & 59 \\
\hline Pneumothorax, not requiring chest tube & 0 & 11 \\
\hline Pneumothorax, requiring chest tube & 0 & 5 \\
\hline \multicolumn{3}{|l|}{ Mediastinoscopy outcomes } \\
\hline No complications & 1 & 11 \\
\hline Pneumothorax, not requiring chest tube & 0 & 1 \\
\hline \multicolumn{3}{|l|}{ Bronchoscopy without TBNA outcomes } \\
\hline No complications & 0 & 48 \\
\hline \multicolumn{3}{|l|}{ Bronchoscopy with EBUS-TBNA outcomes } \\
\hline No complications & 30 & 107 \\
\hline Transient hypoxemia & 0 & 1 \\
\hline
\end{tabular}

See Table 2 legend for expansion of abbreviations. 
Table 4-Diagnostic Yield of EBUS-TBNA Based on CT-PET Scan Nodal Status

\begin{tabular}{|c|c|c|c|c|}
\hline $\begin{array}{l}\text { Lymph Node Stage } \\
\text { by CT-PET Scana }\end{array}$ & $\begin{array}{c}\text { Bronchoscopy } \\
\text { With EBUS-TBNA } \\
\text { Insufficient }\end{array}$ & $\begin{array}{c}\text { EBUS-TBNA Made a Specific } \\
\text { Diagnosis of Lymph } \\
\text { Node Involvement }\end{array}$ & $\begin{array}{c}\text { EBUS-TBNA Did Not Make a Specific Diagnosis } \\
\text { but Other Bronchoscopic Measures Did }\end{array}$ & Total \\
\hline N1 & $11(44)$ & $8(32)$ & $6(24)$ & 25 \\
\hline $\mathrm{N} 2$ & $23(32)$ & $37(52)$ & $11(15)$ & 71 \\
\hline $\mathrm{N} 3$ & $15(37)$ & $22(54)$ & $4(10)$ & 41 \\
\hline Total & $49(36)$ & $67(49)$ & $21(15)$ & 137 \\
\hline
\end{tabular}

Data presented as No. (\%). See Table 2 legend for expansion of abbreviation.

a Lymph node stage based on CT scan and PET imaging. If CT scans and PET scans were discordant, the higher stage was always chosen.

rather than as multiple parts. The real question is how can we both stage and diagnose a patient at the same time, essentially working on multiple questions in parallel rather than in series. Because of this, multiple guidelines recommend biopsy of the mediastinal lymph nodes first in patients with evidence of nodal disease rather than biopsy of the peripheral mass. ${ }^{2,4-9,26}$ However, to our knowledge there have been no direct comparisons of these alternative strategies. This study is the first, to our knowledge, to compare sequencing strategies and to quantify the differences in outcome. It demonstrates that sampling the mediastinum first with EBUS-TBNA first is a better strategy than sampling peripheral lung masses first in patients with suspected T1-3N1-3M0 disease.

Sampling the mediastinum first with EBUS-TBNA is a superior strategy because it provides all of the requisite information needed to guide treatment decisions, whereas sampling peripheral masses does not. However, with recent developments in targeted therapy and molecular analysis, treatment decisions now frequently require information on the EGFR mutation status of the tumor. Thus, the question of how can we simultaneously stage and diagnose disease in a patient with one test needs to be expanded to include consideration of whether that test can obtain sufficient tissue for molecular analysis. Historically, surgical or core needle biopsies were needed because of the large amount of tissue required for mutation analysis. However, as molecular methods have improved, EBUSTBNA has been shown to provide sufficient material

\section{Table 5-Specialty Ordering CT Image-Guided Needle Biopsy Prior to Mediastinal Sampling}

\begin{tabular}{lccr}
\hline \hline Specialty & $\begin{array}{c}\text { All Testing Within } \\
\text { Our Institution }\end{array}$ & Outside Institutions & Total \\
\hline Internal medicine & 0 & $7(13)$ & $7(11)$ \\
Oncology & $6(60)$ & $1(2)$ & $7(11)$ \\
Pulmonary & 0 & $27(51)$ & $27(43)$ \\
Thoracic surgery & $4(40)$ & $2(4)$ & $6(10)$ \\
Unknown & 0 & $16(30)$ & $16(25)$ \\
Total & 10 & 53 & 63 \\
\hline
\end{tabular}

Data are presented as No. (\%). for EFGR mutation testing in $90 \%$ to $96 \%$ of cases, and it compares favorably to CT image-guided core biopsy in this regard. ${ }^{27-30}$ So, EBUS-TBNA can provide all of the requisite information required to guide treatment, including diagnosis, mutation analysis, and staging.

Unfortunately, our data show that guideline-consistent care using a mediastinal sampling first strategy is quite uncommon. This raises the question of whether newer techniques, such as EBUS-TBNA, were available at the referring centers. When approaching this question, it is important to recognize that these are not hospitalto-hospital transfers. Most of these patients are outpatient referrals. Availability of EBUS-TBNA in these cases is a function of regional availability rather than availability at a particular hospital. However, proper use of EBUS-TBNA is a function of not only availability but also health-care provider awareness of availability and appropriate knowledge of when to use it. Whether providers in these locales are aware of regional EBUS-TBNA availability cannot be determined from our study, but it does warrant further investigation, since improving knowledge of availability might improve quality of care. Importantly, lack of availability of EBUS-TBNA is not an appropriate reason to do a CT image-guided biopsy. If EBUS-TBNA is not available locally, referral to another center for testing or consideration of mediastinoscopy, depending on patient preferences for travel, is reasonable.

Finally, proper test sequencing for lung cancer staging is contingent on providers at the point of first contact having sufficient knowledge of the topic so that they can make good decisions on what tests to order. Educational initiatives to address this quality gap will be needed. Our data show that most of the invasive tests were ordered by pulmonologists or internists. The goal must be knowledge dissemination to the right population of providers, since the decision point is occurring near the primary care level. In this case, the population of providers is very broad and must include everyone, from primary care providers to pulmonologists, oncologists, and surgeons. Local tumor boards probably have a role here in disseminating information on evidence-based best practices. 
The message should be kept relatively simple: In patients with suspected lung cancer without evidence of distant metastatic disease, if there is evidence of possible mediastinal disease by CT scan or PET scan, proceed with EBUS-TBNA. This is consistent with the American College of Chest Physicians' third edition lung cancer guidelines. ${ }^{26}$ As such, it is important to remember that these are evidence-based recommendations, but there are always exceptions; sound clinical judgment needs to be applied, which is the case for all guidelines. ${ }^{2,26}$ For example, in patients with severe comorbidities such as dementia that make subsequent treatment untenable, alternative strategies might be warranted, but these should be fairly rare cases.

Since the most common practice pattern was to order a biopsy of the peripheral lung mass using CT image guidance first, it makes sense to involve interventional radiologists as part of the solution. Since all patients undergoing CT image-guided biopsy have CT imaging, there should be little reason to perform a biopsy of a peripheral lesion to rule out lung cancer if there is evidence of mediastinal disease and the mediastinal lymph nodes have not been sampled. If a patient is referred for CT image-guided biopsy, and there is mediastinal lymphadenopathy that has not undergone biopsy, the interventional radiologist should discuss this with the referring physician so that unnecessary procedures can be avoided. This type of solution lends itself to traditional quality improvement and benchmarking programs as well as targeted physician education. Of course, if EBUS-TBNA and bronchoscopic biopsy are unrevealing, then CT image-guided biopsy may be needed. ${ }^{31-33}$

This study adds to the existing body of literature on quality gaps in lung cancer staging ${ }^{10-15}$ by comparing the effectiveness of different testing sequences on outcomes. However, this study has several limitations, so these results should be considered carefully. First, this was a single-center study from a large cancer center. Most of the patients were referred, often from long distances, and as such the population being studied may not be representative of other practices. However, the very large geographic referral base of our institution makes it less likely that local regional variations are contributing to the observed practice patterns, so there is a good chance that these results are generalizable to a fair number of community practices. Since the study was in a cancer center, it is also possible that the prevalence of cancer in this population was higher than in a general practice. However, most of the patients studied had lung masses $>3 \mathrm{~cm}$ in size along with mediastinal adenopathy. The prevalence of malignant disease in lung masses $>3 \mathrm{~cm}$ is $\geq 90 \%$, so although the prevalence of malignancy may be a bit high, it is probably not excessively so. ${ }^{32,34}$
Given these limitations, larger confirmatory studies are certainly warranted to confirm and quantify this quality gap and to identify the determinants that are driving it.

In summary, we found that there is a large quality gap in terms of the proper sequencing of invasive diagnostic tests for lung cancer staging in patients with T1-3N1-3M0 disease. Guideline-consistent care, defined as sampling of the mediastinal lymph nodes first, occurred in only $22 \%$ of patients. Guidelineinconsistent care, defined as sampling of the peripheral mass without sampling of the mediastinum, was most frequently due to ordering a CT image-guided biopsy first. Guideline-inconsistent care resulted in more invasive tests and complications. EBUS-TBNA provided all the information necessary to both diagnose and stage patients in $64 \%$ of cases. In patients with suspected lung cancer without evidence of distant metastatic disease with mediastinal lymphadenopathy by CT scan or PET scan, guideline-consistent care using EBUS-TBNA as the first invasive test will save money and decrease complications.

\section{ACKNOWLEDGMENTS}

Author contributions: Dr Ost is the principal investigator and takes responsibility for the study oversight, data integrity, and analysis of the project as a whole.

Dr Almeida: contributed to data collection, writing, analysis, review, revision, and final approval.

Dr Casal: contributed to the analysis, review, revision, and final approval.

Dr Jimenez: contributed to the analysis, review, revision, and final approval.

Dr Eapen: contributed to the analysis, review, revision, and final approval.

Dr Uzbeck: contributed to the analysis, review, revision, and final approval.

Dr Sarkiss: contributed to the analysis, review, revision, and final approval.

Dr Rice: contributed to the analysis, review, revision, and final approval.

Dr Morice: contributed to the analysis, review, revision, and final approval.

Dr Ost: contributed to data collection, auditing, analysis, writing, review, revision, and final approval.

Financial/nonfinancial disclosures: The authors have reported to CHEST the following conflicts of interest: In the last 3 years, Dr Eapen has received unrestricted educational grants from the Olympus Corporation and has provided consulting services to the Pentax Ricoch Imaging Co, Ltd. Dr Rice is a consultant to Olympus Corporation and teaches courses on EBUS. Drs Almeida, Casal, Jimenez, Uzbeck, Sarkiss, Morice, and Ost have reported that no potential conflicts of interest exist with any companies/organizations whose products or services may be discussed in this article.

Other contributions: All work was performed at The University of Texas MD Anderson Cancer Center, Houston, Texas.

\section{REFERENCES}

1. Silvestri GA, Gould MK, Margolis ML, et al. Noninvasive staging of non-small cell lung cancer: ACCP evidence-based clinical practice guidelines (2nd ed). Chest. 2007;132(3_suppl): 178S-201S. 
2. Silvestri GA, Gonzalez AV, Jantz MA, et al. Methods for staging non-small cell lung cancer: diagnosis and management of lung cancer, 3rd ed: American College of Chest Physicians evidence-based clinical practice guidelines. Chest. 2013(5_suppl): e211S-e250S

3. Gould MK, Kuschner WG, Rydzak CE, et al. Test performance of positron emission tomography and computed tomography for mediastinal staging in patients with non-small-cell lung cancer: a meta-analysis. Ann Intern Med. 2003;139(11): 879-892.

4. Almeida FA, Uzbeck M, Ost D. Initial evaluation of the nonsmall cell lung cancer patient: diagnosis and staging. Curr Opin Pulm Med. 2010;16(4):307-314.

5. Detterbeck FC, Jantz MA, Wallace M, Vansteenkiste J, Silvestri GA. Invasive mediastinal staging of lung cancer: ACCP evidence-based clinical practice guidelines (2nd ed). Chest. 2007;132(3_suppl):202S-220S.

6. Rivera MP, Mehta AC. Initial diagnosis of lung cancer: ACCP evidence-based clinical practice guidelines (2nd ed). Chest. 2007;132(3_suppl):131S-148S.

7. Baldwin DR, White B, Schmidt-Hansen M, Champion AR, Melder AM; Guideline Development Group. Diagnosis and treatment of lung cancer: summary of updated NICE guidance. BMJ. 2011;342:d2110.

8. Crinò L, Weder W, van Meerbeeck J, Felip E; ESMO Guidelines Working Group. Early stage and locally advanced (nonmetastatic) non-small-cell lung cancer: ESMO clinical practice guidelines for diagnosis, treatment and follow-up. Ann Oncol. 2010;21(suppl 5):v103-v115.

9. De Leyn P, Lardinois D, Van Schil PE, et al. ESTS guidelines for preoperative lymph node staging for non-small cell lung cancer. Eur J Cardiothorac Surg. 2007;32(1):1-8.

10. Little AG, Rusch VW, Bonner JA, et al. Patterns of surgical care of lung cancer patients. Ann Thorac Surg. 2005;80(6): 2051-2056.

11. Schipper P, Schoolfield M. Minimally invasive staging of N2 disease: endobronchial ultrasound/transesophageal endoscopic ultrasound, mediastinoscopy, and thoracoscopy. Thorac Surg Clin. 2008;18(4):363-379.

12. Haponik EF, Shure D. Underutilization of transbronchial needle aspiration: experiences of current pulmonary fellows. Chest. 1997;112(1):251-253.

13. Smyth CM, Stead RJ. Survey of flexible fibreoptic bronchoscopy in the United Kingdom. Eur Respir J. 2002;19(3):458-463.

14. Prakash UB, Offord KP, Stubbs SE. Bronchoscopy in North America: the ACCP survey. Chest. 1991;100(6):1668-1675.

15. Colt HG, Prakash UBS, Offord KP. Bronchoscopy in North America: survey by the American Association for Bronchology, 1999. J Bronchol Interv Pulmonol. 2000;7(1):8-25.

16. Yasufuku K, Pierre A, Darling G, et al. A prospective controlled trial of endobronchial ultrasound-guided transbronchial needle aspiration compared with mediastinoscopy for mediastinal lymph node staging of lung cancer. J Thorac Cardiovasc Surg. 2011;142(6):1393-1400.e1.

17. Annema JT, Versteegh MI, Veseliç M, et al. Endoscopic ultrasound added to mediastinoscopy for preoperative staging of patients with lung cancer. JAMA. 2005;294(8):931-936.

18. Ernst A, Anantham D, Eberhardt R, Krasnik M, Herth FJ. Diagnosis of mediastinal adenopathy-real-time endobronchial ultrasound guided needle aspiration versus mediastinoscopy. J Thorac Oncol. 2008;3(6):577-582.

19. Sharples LD, Jackson C, Wheaton E, et al. Clinical effectiveness and cost-effectiveness of endobronchial and endoscopic ultrasound relative to surgical staging in potentially resectable lung cancer: results from the ASTER randomised controlled trial. Health Technol Assess. 2012;16(18):1-75.

20. Al-Sarraf N, Aziz R, Gately K, et al. Pattern and predictors of occult mediastinal lymph node involvement in non-small cell lung cancer patients with negative mediastinal uptake on positron emission tomography. Eur J Cardiothorac Surg. 2008;33(1):104-109.

21. Eapen GA, Shah AM, Lei X, et al. Complications, consequences, and practice patterns of endobronchial ultrasoundguided transbronchial needle aspiration: results of the AQuIRE registry. Chest. 2013;143(4):1044-1053.

22. Yarmus LB, Akulian J, Lechtzin N, et al. Comparison of 21-gauge and 22-gauge aspiration needle in endobronchial ultrasoundguided transbronchial needle aspiration: results of the American College of Chest Physicians Quality Improvement Registry, Education, and Evaluation Registry. Chest. 2013;143(4): 1036-1043.

23. Ost DE, Ernst A, Lei X, et al; AQuIRE Bronchoscopy Registry. Diagnostic yield of endobronchial ultrasound-guided transbronchial needle aspiration: results of the AQuIRE Bronchoscopy Registry. Chest. 2011;140(6):1557-1566.

24. AHRQ. Series overview and methodology. In: Shojania K, McDonald K, Wachter R, Owens DK, eds. Closing the Quality Gap: A Critical Analysis of Quality Improvement Strategies. Rockville, MD: Agency for Healthcare Research and Quality, 2004;1-37.

25. Institute of Medicine (Committee on Quality of Health Care in America). Crossing the Quality Chasm: A New Health System for the 21st Century. Washington, DC: National Academies Press; 2001.

26. Ost DE, Yeung SC, Tanoue LT, Gould MK. Clinical and organization factors in the initial evaluation of patients with lung cancer: diagnosis and management of lung cancer, 3rd ed: American College of Chest Physicians evidence-based clinical practice guidelines. Chest. 2013;143(5_suppl):e121S-e141S.

27. Billah S, Stewart J, Staerkel G, Chen S, Gong Y, Guo M. EGFR and KRAS mutations in lung carcinoma: molecular testing by using cytology specimens. Cancer Cytopathol. 2011; $119(2): 111-117$.

28. Nakajima T, Yasufuku K, Suzuki M, et al. Assessment of epidermal growth factor receptor mutation by endobronchial ultrasound-guided transbronchial needle aspiration. Chest. 2007;132(2):597-602

29. Allegrini S, Antona J, Mezzapelle R, et al. Epidermal growth factor receptor gene analysis with a highly sensitive molecular assay in routine cytologic specimens of lung adenocarcinoma. Am J Clin Pathol. 2012;138(3):377-381.

30. Garcia-Olivé I, Monsó E, Andreo F, et al. Endobronchial ultrasound-guided transbronchial needle aspiration for identifying EGFR mutations. Eur Respir J. 2010;35(2):391-395.

31. Ost DE, Gould MK. Decision making in patients with pulmonary nodules. Am J Respir Crit Care Med. 2012;185(4): 363-372.

32. Ost D, Fein AM, Feinsilver SH. Clinical practice. The solitary pulmonary nodule. $N$ Engl J Med. 2003;348(25): 2535-2542.

33. Grosu HB, Eapen GA, Jimenez CA, Morice RC, Ost D. Lung cancer screening: making the transition from research to clinical practice. Curr Opin Pulm Med. 2012;18(4):295-303.

34. Ost D, Fein A. Evaluation and management of the solitary pulmonary nodule. Am J Respir Crit Care Med. 2000;162(3 pt 1): 782-787. 